\title{
Analysis of diffusion process in fractured reservoirs using fractional derivative approach
}

\author{
Kambiz Razminia, Abolhassan Razminia, J.A. Tenreiro Machado
}

\begin{abstract}
The fractal geometry is used to model of a naturally fractured reservoir and the concept of fractional derivative is applied to the diffusion equation to incorporate the history of fluidflow in naturally fractured reservoirs. The resulting fractally fractional diffusion (FFD) equation is solved analytically in the Laplace space for three outer boundary conditions. The analytical solutions are used to analyze the response of a naturally fractured reservoirconsidering the anomalous behavior of oil production. Several synthetic examples are provided to illustrate the methodology proposed in this work and to explain the diffusionprocess in fractally fractured systems.
\end{abstract}

Keywords:

Fractal geometry, Naturally fractured reservoir Fractional derivative, FFD model

1. Introduction

It is well known that the flow distribution and propagation within naturally fractured reservoirs (double porosity systems) is controlled mostly by the distribution of fractures [1]. The presence of fractures at different scales represents an element of uncertainty and heterogeneity in the construction of a reservoir model [1,2]. Due to this reason, classical Euclidean models cannot describe the complexities of such systems. Alternatively, fractal theory provides a powerful method to describe the complex network of fractures [1-4].

Chang and Yortsos [5] were the first authors adopting a fractal model to describe the response of a double porosity system (naturally fractured reservoir). Acuña et al. [6] applied this model to naturally fractured reservoirs and found that the wellbore pressure is a power-law function of time. Flamenco-Lopez and Camacho-Velazquez [7] discussed two approaches to describe and analyze a naturally fractured reservoir with fractal geometry: (1) analysis of both transient and pseudo- steadystate flow periods of well pressure tests, (2) determination of fractal-model parameters from porosity well logs or from another source $[1,8]$.

From another point of view, fractional calculus $(F C)$ as the generalization of the ordinary calculus, attracted the attention of scientists from many disciplines. Capturing the memory of a dynamical phenomenon via integer-order calculus poses dif- ficulties, while FC by introducing a kernel considers inherently the hereditary of complex processes. Specifically, in the last two decade petroleum engineering has been impressed by FC. Several authors used the concept of FC to describe the complexity of diffusion process in porous media [9-11]. These researchers developed a mathematical model to define the response of oil reservoirs. 
Nomenclature

$A_{w b} \quad$ wellbore area, $\mathrm{ft}^{3}\left(\mathrm{~L}^{3}\right)$

$a V_{s} \quad$ fractal parameter related to the porosity of the fracture network, $\mathrm{L}_{m f}^{3-d}$

$B \quad$ oil formation volume factor, RB/STB $\left(\mathrm{L}^{3} / \mathrm{L}^{3}\right)$

C compressibility, $\mathrm{psi}^{-1}\left(\mathrm{~L} \mathrm{t}^{2} / \mathrm{m}\right)$

$C_{s} \quad$ wellbore storage coefficient, bbl/psi $\left(\mathrm{L}^{4} \mathrm{t}^{2} / \mathrm{m}\right)$

$C_{D} \quad$ dimensionless wellbore storage coefficient

d Euclidean dimension

$d_{m f} \quad$ mass fractal dimension

$g \quad$ acceleration of gravity, $\mathrm{ft} / \mathrm{s}^{2}\left(\mathrm{~L} / \mathrm{t}^{2}\right)$

$g_{c} \quad$ gravitational units conversion factor, $32.17\left(\mathrm{lb}_{\mathrm{m}} / \mathrm{ft}\right) /\left(\mathrm{lb}_{\mathrm{f}} \mathrm{s}^{2}\right)$

$h \quad$ formation thickness, $\mathrm{ft}(\mathrm{L})$

I modified Bessel function of the first kind

$k \quad$ permeability, md $\left(\mathrm{L}^{2}\right)$

$K \quad$ modified Bessel function of the second kind

$L \quad$ Laplace transform of

$m \quad$ fracture-network parameter, $\mathrm{L}^{2 \mathrm{p} h}$

$p \quad$ pressure, psi $\left(\mathrm{m} /\left(\mathrm{Lt}^{2}\right)\right)$

$p_{D w} \quad$ dimensionless wellbore pressure for the constant-rate case without wellbore storage and skin effects

$p_{w D} \quad$ dimensionless wellbore pressure with wellbore storage and skin effects

$p_{w f} \quad$ wellbore flowing pressure, psi $\left(\mathrm{m} /\left(\mathrm{Lt}^{2}\right)\right)$

$q \quad$ production oil rate, STB/D $\left(\mathrm{L}^{3} / \mathrm{t}\right)$

$q_{D} \quad$ dimensionless rate

$r \quad$ radial distance, $\mathrm{ft}(\mathrm{L})$

$r_{D} \quad$ dimensionless radial distance

$S \quad$ total skin factor

$t \quad$ time, hours ( $\mathrm{t}$ )

$t_{D} \quad$ dimensionless time

$u \quad$ Laplace transform variable

c fractional derivative order

C gamma function

$h \quad$ conductivity index

$k \quad$ interporosity flow parameter

$\boldsymbol{l} \quad$ oil viscosity, cp (m/(Lt))

$q$ density of liquid in the wellbore, $\mathrm{lb}_{\mathrm{m}} / \mathrm{ft}^{3}\left(\mathrm{~m} / \mathrm{L}^{3}\right)$

$r \quad$ matrix/fracture interaction index

$\nearrow \quad$ porosity

$x \quad$ storativity ratio

Subscripts

D dimensionless

e external

$f \quad$ fracture (fissure)

m matrix

$w \quad$ wellbore

It is generally accepted that the diffusion process is history-dependent in fractal systems. In fractally fractured reservoirs, history of flow and nonlocality are pivotal in all stages of production. Metzler et al. [9] applied a fractional derivative approach to fractal model to incorporate the memory.

To characterize a naturally fractured reservoir, it is required the estimation of the fractal and fractional parameters (dimensionless parameters) of the mathematical model. In spite of all the work done on fractally fractional diffusion (FFD), an appropriate analytical solution has not been addressed in the literature to calculate the fractal and fractional parameters and to analyze the effects of these parameters upon reservoir behavior. This work presents an analytical solution for the pressure response of naturally fractured reservoirs during transient and boundary-dominated flow periods, in the light of FC. In particular, the response of fractured systems is investigated on the basis of fractional derivative. The analytical solutions are discussed by several synthetic examples.

This paper is organized as follows. Section 2 introduces the fundamentals of fractional calculus. Sections 3 and 4 develop and introduce the FFD model and some synthetic examples, respectively. Finally, the concluding remarks are discussed in Section 5. 


\section{Fractional calculus}

If we define the Gamma function as $\Gamma(q)=\int_{0}^{\infty} e^{-z} z^{q-1} d z, q>0$, then the fractional order integral of a Lebesgue integrable function $x(t)$ can be defined as [12]:

$$
{ }_{a} D_{t}^{-q} x(t):=\frac{1}{\Gamma(q)} \int_{a}^{t}(t-\tau)^{q-1} x(\tau) d \tau, \quad q \in \mathbf{R}^{+} .
$$

Based on the fractional integral definition, the fractional order derivative in the sense of Riemann-Liouville (RL) can be defined as follows:

$$
{ }_{a}^{R 1} D_{t}^{q} x(t):=D^{m}{ }_{a} D_{t}^{-(m-q)} x(t)=\frac{1}{\Gamma(m-q)} \frac{d^{m}}{d t^{m}} \int_{a}^{t}(t-\tau)^{m-q-1} x(\tau) d \tau, \quad m-1<q<m \in \mathbf{Z}^{+} .
$$

Remark 1 [13]. For the fractional derivative and integral RL operators we have:

1. $\left\{L\left\{{ }^{a} D_{t}^{-q} x(t)\right\}=u-q X(u)\right.$,

2. $\lim _{q \rightarrow m}^{0} D_{t}^{-q} x(t)={ }^{0} D_{t}^{-m} x(t), q>0, m \in \mathbf{Z}^{+}$,

3. $\frac{p t}{0} D_{t}^{q} c=\frac{d q-1}{\Gamma(1-q)}$,
4. $\left.L_{0}^{\{t} D_{t}^{q} X(t)\right\}=u^{q} X(u)-\sum_{k=0}^{m-1} u^{k} \cdot \frac{p l t}{0} D_{t}^{q-k-1} x(0), m-1<q<m \in \mathbf{Z}^{+}$.

The RL derivative of a constant is not zero; moreover, its Laplace transform needs fractional derivatives of the function in initial time.

Definition 1 (see, e.g. [12,14]). The fractional order derivative operator in the sense of Caputo (C) is defined as follows:

$$
{ }_{a}^{C} D_{t}^{f} x(t):={ }_{a}^{R t} D_{t}^{-(m-q)} D^{m} x(t)=\frac{1}{\Gamma(m-q)} \int_{a}^{t}(t-\tau)^{m-q-1} x^{(m)}(\tau) d \tau, \quad m-1<q<m \in \mathbf{Z}^{+} .
$$

Remark 2 [13]. For the fractional Caputo derivative operator we have:

1. ${ }_{0}^{c} D_{t}^{q} \mathrm{c}=0$

2. ${ }_{0}^{C} D_{t}^{q}{ }_{0} D_{t}^{-q} x(t)=0_{\text {gLt }}^{D Q}{ }_{0} D_{t}^{-q} x(t)=x(t), 0<q<1$,

3. $L\left\{{ }_{0}^{c} D_{t}^{q} X(t)\right\}=u^{q} X(u)-\sum_{k=0}^{m-1} u^{q-k-1} x^{(k)}(0), m-1<q<m \in \mathbf{Z}^{+}$.

$\xi$

For this type of fractional derivative the Laplace transform needs integer order derivatives in the initial times for the func- tion. In addition, the Caputo derivative of a constant is zero, which is consistent to the ordinary calculus. For more informa- tion about FC, one can refer to $[15,16]$.

\section{Analysis of FFD model}

In this section the basis of FFD model and its analytical solution are presented. Firstly, the properties and concepts of nat- urally fractured reservoirs are analyzed in a mathematical model (FFD model). The required dimensionless parameters are defined. Then the analytical solution of the FFD model is given in Laplace space for three different outer boundary conditions.

\subsection{Mathematical model}

Two distinct porous media are interacting in a fractured reservoir: the matrix blocks, with high storativity and low permeability, and the fractures (fissures) system, with low storativity and high permeability. When a well is opened in a frac- tured reservoir, a rapid pressure response occurs in the fracture network due to its high diffusivity. A pressure difference is created between matrix and fracture, and the matrix blocks start to flow into the fractures. The pressure of the matrix blocks $p_{m}$ decreases as flow progresses and, finally, tends to equalize with the pressure of the surrounding fractures $p_{f}$ (Figs. 1 and

2). Before discussing the mathematical model of such systems, the related dimensionless variables are defined in Table 1.

The storativity ratio defines the contribution of the fissure system to the total storativity. $a V_{s}$ for a cylindrical symmetry is given by:

$$
2 \pi h \phi_{f}(r)=a V_{s} r^{d-s_{j}-d}
$$

By setting $d_{m f} 1 / 4 d 1 / 42$, the typical Warren and Root [17] storativity ratio can be obtained. 


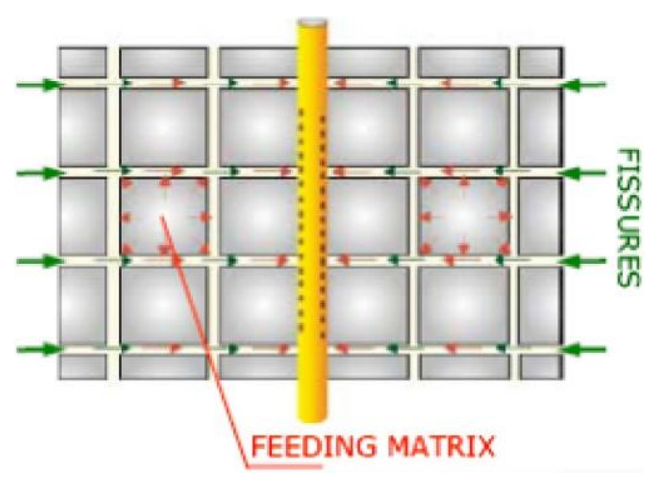

Fig. 1. Schematic diagram of naturally fracture reservoir (Euclidean).

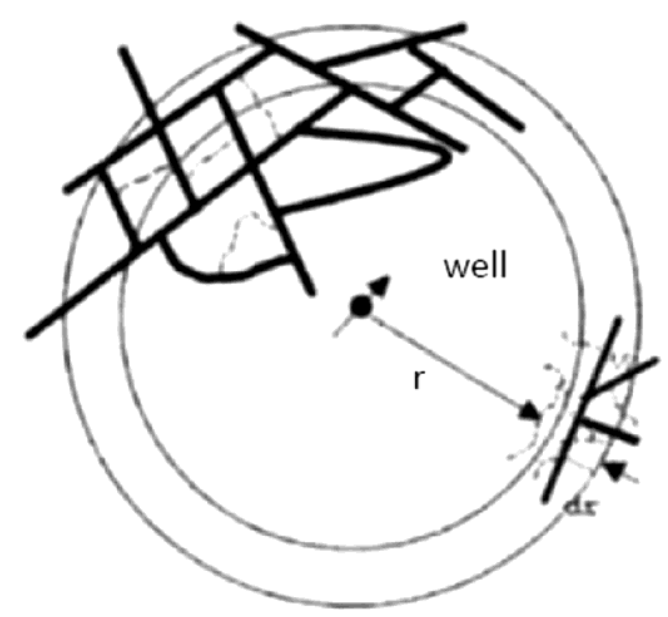

Fig. 2. Schematic diagram of naturally fracture reservoir with fractal behavior (Park et al. [10]).

Table 1

Dimensionless variables.

Dimensionless pressure

Storativity ratio

Interporosity flow coefficient

Dimensionless time

Dimensionless radius

Dimensionless wellbore storage

Total skin factor

$$
\begin{aligned}
& P_{D}=\frac{k_{t}}{\text { का. } 2 \text { काp }}\left(p_{i}-p(r, r)\right)
\end{aligned}
$$

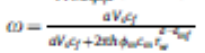

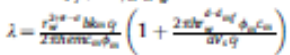

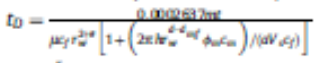

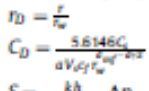

$$
\begin{aligned}
& S=\frac{k b}{141.24 \mu} \Delta p
\end{aligned}
$$

The interporosity flow coefficient is used to describe the ability of the matrix blocks to flow into the fissures. The coefficient $e$ is a parameter for distance from matrix to fracture. The parameter $m$ is the local structural property of the fractal network, similar to the conventional Euclidean permeability that is defined as follows:

$$
k_{f}(r)=\left(\frac{a V_{s}}{2 \pi h}\right) m r^{d-d-d-\theta}
$$

The wellbore storage coefficient $C_{s}$ is given by:

$$
C_{s}=\frac{144 A_{\text {vb }}}{5.615 \rho} \frac{g_{c}}{g}
$$


where $A_{w b}$ is the wellbore area, $q\left(\mathrm{lb}_{\mathrm{m}} / \mathrm{ft}^{3}\right)$ denotes the density of the liquid (oil) in the wellbore and $g=g$ $=\mathrm{lb}_{\mathrm{f}} / \mathrm{lb}_{\mathrm{m}}$.

Chang and Yortsos [5] presented a generalization of the Warren and Root [14] model to describe a double porosity fractal system (dimensionless form):

$$
\frac{1}{r_{D}^{g}} \frac{\partial^{2} p_{D f}}{\partial r_{D}^{2}}+\frac{\beta}{r_{D}^{g}+1} \frac{\partial p_{D f}}{\partial r_{D}}=\omega \frac{\partial p_{D f}}{\partial t_{D}}+(1-\omega) r_{D}^{d-d m} \frac{\partial p_{D m}}{\partial t_{D}}
$$

Applying the same approach used by Metzler et al. [9], based on the concepts of fractal geometry and FC, the dimension- less FFD model in naturally fractured reservoirs can be written as:

$$
\frac{1}{r_{D}^{g}} \frac{\partial^{2} p_{D f}}{\partial r_{D}^{2}}+\frac{\beta}{r_{D}^{g+1}} \frac{\partial p_{D f}}{\partial r_{D}}=\omega \frac{\partial^{\gamma} p_{D f}}{\partial t_{D}^{\gamma}}+(1-\omega) r_{D}^{d-d_{m f}} \frac{\partial^{\gamma} p_{D m}}{\partial t_{D}^{\gamma}}
$$

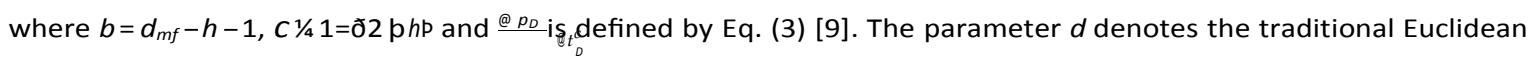
dimension. The parameter $d_{m f}$ is the fractal topological dimension of the system; and the index $h$ is the anomalous diffusion exponent (or the dynamic fractal dimension), which is greater than zero in general (so $0<c 61$ ). Equation (5) reduces to the traditional Euclidean diffusivity equation when $d_{m f} 1 / 4 d 1 / 42, h 1 / 40$ and $c \frac{1}{4} 1$. Therefore Eq. (5) can be considered as a generalization of the classical diffusion equation with respect to the first order time derivative.

According to Chang and Yortsos [5], the mathematical representation of the transfer of fluid from matrix to fracture is:

$$
\frac{\partial p_{D \mathrm{~m}}}{\partial t_{D}}=\frac{\lambda}{r_{D}^{\sigma}}\left(p_{D f}-p_{D \mathrm{~m}}\right)
$$

where $r$ is the matrix/fracture interaction index.

The initial and boundary conditions are presented in Table 2.

The sensitivity of pressure response to fractal dimension is weak [18]. So, it can be assumed that $d_{m f}=d$. Then Eq. (5) reduces to the following relation:

$$
\frac{1}{r_{D}^{\beta}} \frac{\partial^{2} p_{D f}}{\partial r_{D}^{2}}+\frac{\beta}{r_{D}^{\beta+1}} \frac{\partial p_{D f}}{\partial r_{D}}=\omega \frac{\partial^{\gamma} p_{D f}}{\partial t_{D}^{\gamma}}+(1-\omega) \frac{\partial^{\gamma} p_{D m}}{\partial^{\gamma} t_{D}}
$$

Based on the same approach given by Metzler et al. [9], the fractional form of differential equation for the transfer of fluid from matrix to fracture under restricted flow condition (the matrix response is slower) can be written as:

$$
\frac{\partial^{7} p_{D \mathrm{~m}}}{\partial^{7} t_{D}}=\frac{\lambda}{(1-\omega)}\left(p_{D f}-p_{D m}\right)
$$

When $c 1 / 4$ 1, Eq. (8) reduces to the corresponding Warren and Root [14] equation.

\subsection{Analytical solution}

Using the initial conditions, Eqs. (7) and (8) can be written in Laplace space as:

$$
\frac{1}{r_{D}^{\beta}} \frac{\partial^{2} \bar{p}_{D f}}{\partial r_{D}^{2}}+\frac{\beta}{r_{D}^{s+1}} \frac{\partial \bar{p}_{D f}}{\partial r_{D}}=\omega u^{y} \bar{p}_{D f}+(1-\omega) u^{\gamma} \bar{p}_{D m}
$$

and

$$
u^{\gamma} \bar{p}_{D m}=\frac{\lambda}{(1-\omega)}\left(\bar{p}_{D f}-\bar{p}_{D m}\right)
$$

where $u$ is the Laplace variable.

Combining Eqs. (9) and (10) yields

$$
r_{D}^{2} \frac{\partial^{2} \bar{p}_{D f}}{\partial r_{D}^{2}}+\beta r_{D} \frac{\partial \bar{p}_{D f}}{\partial r_{D}}=r_{D}^{(2+\theta)} u^{\gamma}\left(\frac{\omega(1-\omega) u^{\gamma}+\lambda}{(1-\omega) u^{\gamma}+\lambda}\right) \bar{p}_{D f}
$$



Table 2

Initial and boundary conditions

\begin{tabular}{|c|c|c|c|}
\hline Condition & Infinite (3rd row) & Bounded (3rd row) & Constant pressure (3rd row) \\
\hline $\begin{array}{l}\text { Initial (fracture and matrix) } \\
\text { Inner boundary }\end{array}$ & $\begin{array}{l}p_{Q r}\left(r_{D}, 0\right)=0 \\
\left(r_{D}^{k} \partial p_{\Delta r} / \partial r_{D}\right)_{r_{a}=1}=-1\end{array}$ & $P_{D m}\left(r_{D}, 0\right)=0$ & \\
\hline Outer boundary & $\lim _{r_{a} \rightarrow \infty} p_{\Delta r}\left(r_{D}, t_{D}\right)=0$ & $\left(\partial p_{D} / \partial r_{D}\right)_{r_{a}=r_{a}}=0$ & $\operatorname{par}\left(t_{e D}, t_{D}\right)=0$ \\
\hline
\end{tabular}

Eq. (11) can be simplified to the following formula:

$$
r_{D}^{2} \frac{\partial^{2} \bar{p}_{D f}}{\partial r_{D}^{2}}+\beta r_{D} \frac{\partial \bar{p}_{D f}}{\partial r_{D}}=r_{D}^{(2+\theta)} u^{y} f(u) \bar{p}_{D f}
$$

where

$$
f(u)=\frac{\omega(1-\omega) u^{\gamma}+\lambda}{(1-\omega) u^{\gamma}+\lambda}
$$

Expression (11) can be reduced to Bessel's equation with the following substitutions:

$$
\begin{aligned}
& \quad p_{D f}=r_{D}^{(1-\beta) / 2} W \\
& \text { and } \\
& \frac{2 \sqrt{f(u)} u^{y / 2}}{2+\theta} r_{D}^{(2+\theta) / 2}=x
\end{aligned}
$$

After substituting, the final form of Eq. (11) can be written as:

$$
x^{2} \frac{\partial^{2} W}{\partial x^{2}}+x \frac{\partial W}{\partial x}-\left(x^{2}+v^{2}\right) W=0
$$

where $v=\frac{1-\beta}{2+0}$.

The general solution of Eq. (15) can be obtained based on the theory of modified Bessel functions:

$$
\bar{W}\left(r_{D}, u\right)=A I_{v}(x)+B K_{v}(x)
$$

where $I_{m}$ and $K_{m}$ are the modified Bessel functions of the first and second kind of order $m 2 R$.

By setting Eqs. (13) and (14) into Eq. (16), the dimensionless pressure drop in Laplace space can be obtained by the following formula:

$$
\bar{p}_{D f}\left(r_{D}, u\right)=r_{D}^{(1-\tilde{\rho}) / 2}\left[A I_{v}\left(\sqrt{f(u)} \frac{2 u^{y / 2}}{2+\theta} r_{D}^{(2+\theta) / 2}\right)+B K_{v}\left(\sqrt{f(u)} \frac{2 u^{y / 2}}{2+\theta} r_{D}^{(2+\theta) / 2}\right)\right]
$$

Equation (17) can be abbreviated to the following relation:

$$
\bar{p}_{D f}\left(r_{D}, u\right)=r_{D}^{(1-\tilde{\rho}) / 2}\left[A I_{v}\left(\sqrt{f(u)} g\left(r_{D}, u\right)\right)+B K_{v}\left(\sqrt{f(u)} g\left(r_{D}, u\right)\right)\right]
$$

where the function $g$ is defined as:

$$
g\left(r_{D}, u\right)=\frac{2 u^{y / 2}}{2+\theta} r_{D}^{(2+\theta) / 2}
$$


By making use of the van Everdingen and Hurst [19] method, the solution in Laplace space to a variable flowing bottomhole pressure at a constant rate is $p_{D w} \partial u \mathrm{P} 1_{4}^{1 / 4}{ }^{1} \frac{}{u^{2} q_{D}}$; where $q_{D}$ is the variable rate at constant flowing bottom-hole pressure.

For a constant bottom-hole pressure, the inner boundary condition in Laplace space can be written as:

$$
\bar{p}(1, u)=\frac{1}{u}
$$

The particular solutions of Eq. (17) are obtained under one inner boundary condition and three outer boundary conditions assuming the well is producing at a constant rate. Firstly, the boundary conditions of infinite reservoirs are applied to obtain the dimensionless wellbore pressure response. Then the bounded circular reservoirs are analyzed under boundary conditions to find the dimensionless wellbore pressure response. Finally, the boundary conditions of constant pressure outer boundary reservoirs are used to compute the dimensionless wellbore pressure response.

\subsubsection{Infinite reservoir case}

Applying the boundary conditions of infinite reservoirs to Eq. (17) yields

$$
\bar{p}_{D f}\left(r_{D}, u\right)=r_{D}^{(1-\tilde{\rho} / 2} K_{v}\left(g\left(r_{D}, u\right) \sqrt{f(u)}\right) /\left(u K_{v}(g(1, u) \sqrt{f(u)})\right)
$$

This solution is obtained using the following fact:

$$
I_{y}(x \rightarrow \infty)=\infty
$$

And the dimensionless variable rate is obtained by: 


$$
\begin{aligned}
& \bar{q}_{D}=-\left(r_{D}^{f} \frac{\partial \bar{p}_{D f}}{\partial r_{D}}\right)_{r_{D}-1}=\sqrt{f(u)} K_{v-1}(g(1, u) \sqrt{f(u)}) /\left(u^{(1-\gamma / 2)} K_{v}(g(1, u) \sqrt{f(u)})\right) \\
& 2 \\
& 0 \\
& \text { p }
\end{aligned}
$$

Dimensionless wellbore pressure with wellbore storage and skin effects can be obtained by means of the superposition principle (Duhamel's principle) that is presented by van Everdingen and Hurst [16] and Agarwal et al. [20]:

$$
p_{w D}\left(t_{D}\right)=\int_{0}^{t_{D}}\left[\left(1-C_{D} \frac{d p_{w D}(\tau)}{d \tau}\right) \frac{d p_{D w}\left(t_{D}-\tau\right)}{d\left(t_{D}-\tau\right)}\right] d \tau+S\left(1-C_{D} \frac{d p_{w D}\left(t_{D}\right)}{d t_{D}}\right)
$$

where

$p_{w D}\left(t_{D}\right)=$ dimensionless wellbore pressure with wellbore storage and skin effects.

$p_{D w}\left(t_{D}\right)=$ dimensionless wellbore pressure for the constant-rate case without wellbore storage and skin effects.

Applying the Laplace transformation to Eq. (21) yields

$$
\bar{p}_{w D}(u)=\frac{u \bar{p}_{D w}(u)+S}{u\left(1+C_{D} u^{2} \bar{p}_{D w}(u)+C_{D} S u\right)}
$$

$\mathrm{r}$

$$
\bar{p}_{w D}(u)=\frac{1 /\left(u \bar{q}_{D}(u)\right)+S}{u\left(1+C_{D} / \bar{q}_{D}(u)+C_{D} S u\right)}
$$

The inverse transform of Eqs. (22) and (23) can be obtained by the Stehfest [21] numerical algorithm.

\subsubsection{Constant pressure outer boundary case}

The particular solution of Eq. (17) for the constant pressure outer boundary case is:

$$
\bar{p}_{D}\left(r_{D}, u\right)=r_{D}^{(1-\beta) / 2}\left(\frac{-K_{v}\left(g\left(r_{e D}, u\right) \sqrt{f(u)}\right) I_{v}\left(g\left(r_{D}, u\right) \sqrt{f(u)}\right)+I_{v}\left(g\left(r_{e D}, u\right) \sqrt{f(u)}\right) K_{v}\left(g\left(r_{D}, u\right) \sqrt{f(u)}\right)}{u\left(-K_{v}\left(g\left(r_{e D}, u\right) \sqrt{f(u)}\right) I_{v}(g(1, u) \sqrt{f(u)})+I_{v}\left(g\left(r_{e D}, u\right) \sqrt{f(u)}\right) K_{v}(g(1, u) \sqrt{f(u)})\right)}\right)
$$

Eq. (26) has been derived by applying the inner and outer boundary conditions.

Thus, the dimensionless variable rate is:

$$
\bar{q}_{D}=-\left(r_{D}^{s} \frac{\partial \bar{p}_{D}}{\partial r_{D}}\right)_{r_{D}-1}=\frac{\sqrt{f(u)}\left(K_{v}\left(g\left(r_{e D}, u\right) \sqrt{f(u)}\right) I_{v-1}(g(1, u) \sqrt{f(u)})+I_{v}\left(g\left(r_{e D}, u\right) \sqrt{f(u)}\right) K_{v-1}(g(1, u) \sqrt{f(u)})\right)}{u^{(1-y / 2)}\left(-K_{v}\left(g\left(r_{e D}, u\right) \sqrt{f(u)}\right) I_{v}(g(1, u) \sqrt{f(u)})+I_{v}\left(g\left(r_{e D}, u\right) \sqrt{f(u)}\right) K_{v}(g(1, u) \sqrt{f(u)})\right)}
$$

At the end, the dimensionless wellbore pressure values with wellbore storage and skin effects can be obtained from Eq. 23).

4. Illustrative examples

We introduce the following dimensionless groups to plot the analytical solutions in a log-log coordinate system:

$$
\begin{aligned}
& x-\text { axis : }\left(t_{D} / C_{D}\right) a \\
& y \text {-axis : }\left(p_{w D}\right) a \text { and }\left(p_{w D}^{\prime} t_{D}\right) a
\end{aligned}
$$




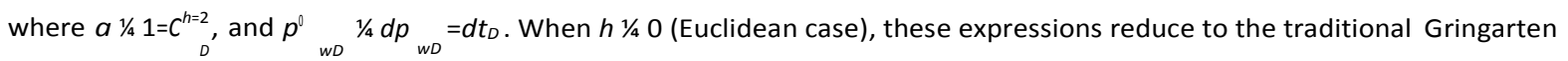
et al. [22] definitions.

The examples are analyzed for dimensionless wellbore storage coefficient $C_{D} 1 / 4100$ and skin factor $S \frac{1}{4} 1: 5$. The solid curves represent the pressure responses, and logarithmic derivatives of pressures are shown by dotted curves. The parameters $h$ and $c$ are shown by theta and gamma in the charts, respectively. Fig. 3 shows the dimensionless wellbore pres- sure drop, Eqs. (20) and (23), and its logarithmic derivative for a reservoir with infinite acting behavior. The effects of con- ductivity index on pressure response, Eqs. (23) and (25), and its logarithmic derivative for bounded circular reservoir are shown in Fig. 4. Fig. 5 presents the response, Eqs. (23) and (27), of dual porosity reservoirs with constant pressure outer boundary condition. These examples are analyzed with three different values of conductivity index $h \frac{1}{4} \mathrm{f0} ; 0: 05 ; 0: 1 \mathrm{~g}$

Jf $1 / 41 ; 0: 9756 ; 0: 9524 g$ p. The results of Figs. 3-5 show that the pressure value and its logarithmic derivative in log-log scale increase with the increase of $h$. The infinite reservoirs (Fig. 3) and finite reservoirs (Figs. 4 and 5) have the same behav- ior before the start of outer boundary effects in finite reservoirs. The main difference between bounded circular reservoir case (Fig. 4) and constant pressure outer boundary case (Fig. 5) is in the effects of outer boundaries. Fig. 4 shows that when the sealing boundary is reached both the pressure and the derivative curve follow a unit slope straight line. Fig. 5 shows that if the side acts as a constant pressure boundary, due to water drive support, the log-log pressure curve tends to stabilize and the derivative drops. The major difference between the fractional and integer order responses indicates that diffusion is slower in reservoirs included memory (considered the history of flow).

Under restricted flow condition, the pressure response of a naturally fractured reservoir in an Euclidean model indicates three different regimes:

(1) At early times only the fissures flow into the well. The contribution of the matrix is negligible. This corresponds to the homogeneous behavior of the fissure system.

(2) At intermediate times the matrix starts to produce into the fissures until the pressure tends to stabilize. This corresponds to a transition flow regime.

(3) Later, the matrix pressure equalizes the pressure of the surrounding fissures. This corresponds to the homogeneous behavior of the total system (matrix and fissures). Then the effect of outer boundaries may be reached.

It should be noted that the depth of the characteristic valley is a function of the transition duration. For small values of $x$, long transition regimes correspond to deep valleys on derivative. The interporosity flow coefficient determines the end of transition period, and the start of the equivalent homogeneous total system flow regime. For smaller $k$ values, the total sys- tem flow starts later. In Figs. 3-5, three curves are generated for $h$ 1/4 f0; 0:05; 0:1g. In the pressure curves and their deriva- tives, the pressure values of larger $h$ and their derivative occurs at higher amplitudes. Furthermore, the smaller the $h$, the deeper the depth of the valley, and the later the start of total system flow (Figs. 3-5), and subsequently the later the effect of outer boundaries in finite reservoirs (Figs. 4 and 5). Fig. 3 reveals that the behavior of total system flow may not be con- sidered as homogeneous in fractally fractured reservoirs, because the derivative slope for larger values of $h$ is not constant

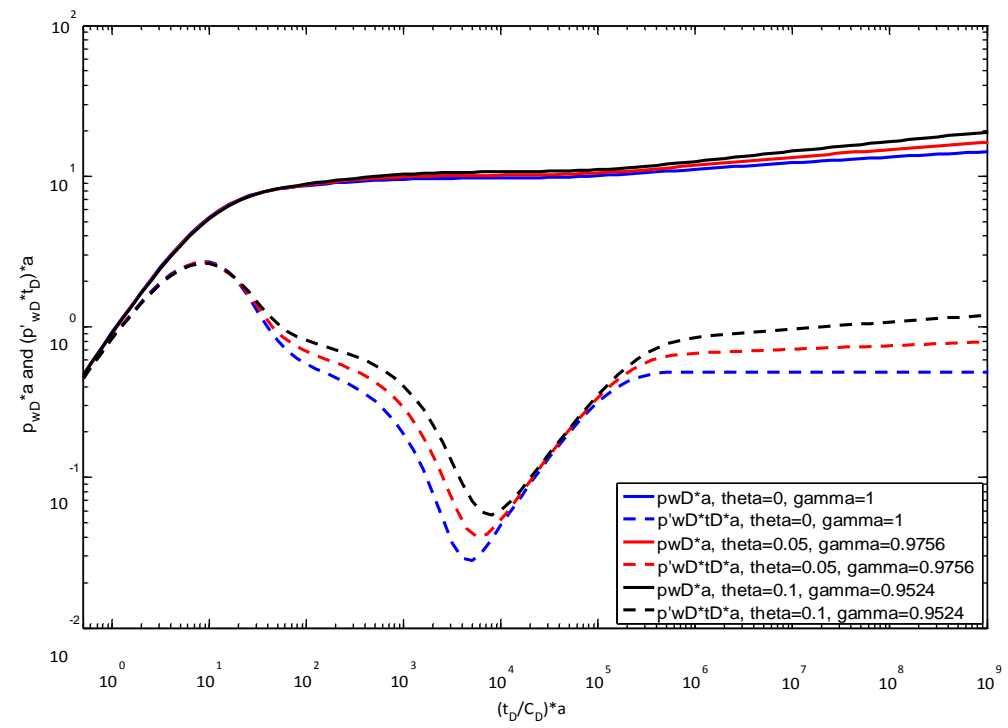

Fig. 3. Solutions of FFD model for fractured reservoirs in an infinite radial system where $X 1 / 40: 01 ; k 1 / 410^{-7}$. 


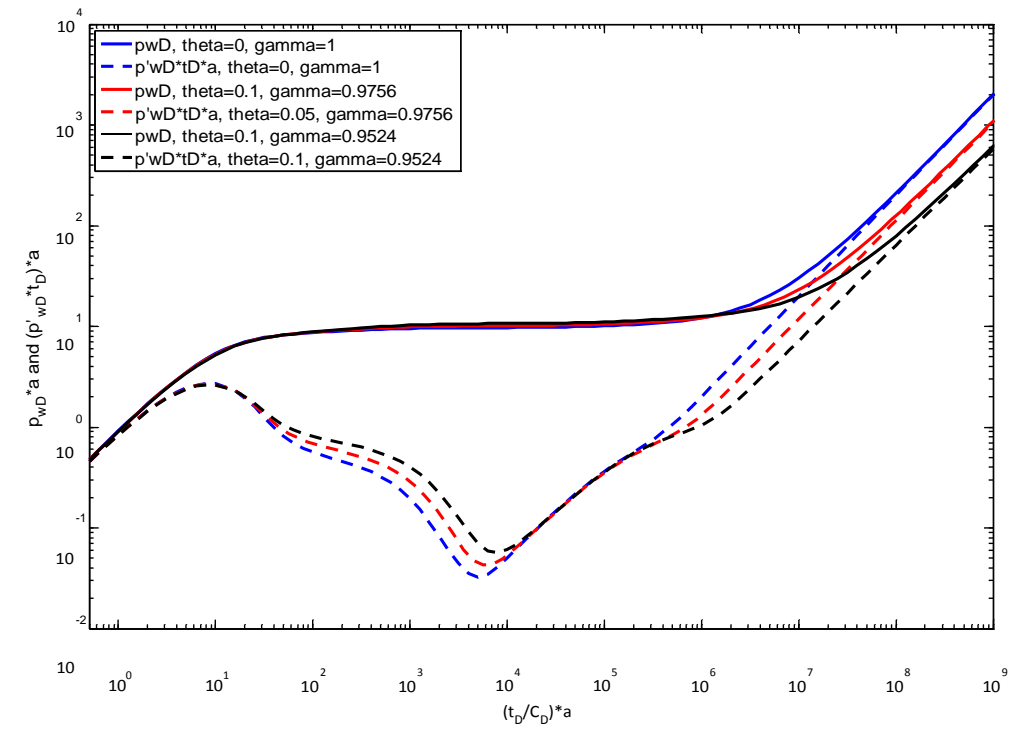

Fig. 4. Solutions of FFD model for fractured reservoirs under no-flow outer boundary condition where $X^{1 / 4} 0: 01 ; k^{1 / 4} 10^{-7}$.

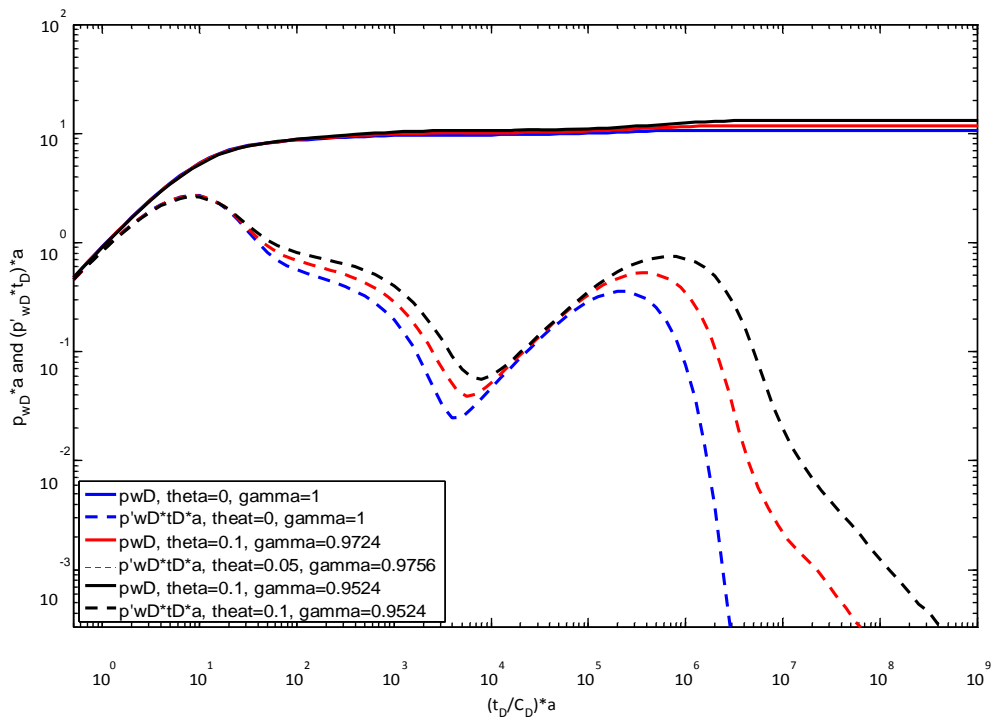

Fig. 5. Solutions of FFD model for fractured reservoirs under constant pressure outer boundary condition where $X^{1 / 4} 0: 01 ; k 1 / 410^{-7}$.

during the times after transition flow regime. As can be seen, the pressure and its logarithmic derivative are parallel straight lines in the times after transition period, which implies that the wellbore pressure is a power law function of time.

In conclusion, we cannot use the conventional methods (semi-log approach and log-log type curves analysis) to interpret transient-pressure data with fractal behavior. Thus, it allows interpreting transient pressure data more accurately with the new dynamical responses.

\section{Concluding remarks}

We synthesized the theory of Bessel's equation and techniques of Laplace transformation to obtain the analytical solution of FFD model assuming the well is flowing at a constant rate. The results of presented examples, based on the analytical solu- tions, reveal that the different values of conductivity index causes some changes in reservoir pressure response. This shows that the traditional Euclidean model cannot be used to analyze fractured reservoirs when oil production exhibits anomalous behavior. The fractional calculus thus provides a powerful tool to improve pressure-transient-test interpretation. 


\section{References}

[1] Camacho-Velázquez R, Fuentes-Cruz G, Vásquez-Cruz M. Decline-curve analysis of fractured reservoirs with fractal geometry. SPE Reserve Eval Eng 2008;11:606-19.

[2] Aasum Y, Kelkar MG, Gupta SP. An application of geostatistics and fractal geometry for reservoir characterization. SPE Formation Eval 1991;6:11-9.[3] Raghavan R, Chen C. Fractured-well performance under anomalous diffusion. SPE Reserve Eval Eng 2013;16:237-45.

[4] Cossio M, Moridis GJ, Blasingame TA. A semianalytic solution for flow in finite-conductivity vertical fractures by use of fractal theory. SPE J 2013;18:83-96.

[5] Chang J, Yortsos YC. Pressure-transient analysis of fractal reservoirs. SPE Formation Eval 1990;5:31-8.

[6] Acuna JA, Ershaghi I, Yortsos Y. Practical application of fractal pressure-transient analysis in naturally fractured reservoirs. SPE Formation Eval 1995;10:173-9.

[7] Flamenco-Lopez F, Camacho-Velazquez R. Determination of fractal parameters of fracture networks using pressure-transient data. SPE Reserve Eval Eng 2003;6:39-47.

[8] Kim TH, Schechter DS. Estimation of fracture porosity of naturally fractured reservoirs with no matrix porosity using fractal discrete fracture networks. SPE Reserve Eval Eng 2009;12:232-42.

[9] Metzler R, Glöckle WG, Nonnenmacher TF. Fractional model equation for anomalous diffusion. Physica A 1994;211:13-24.

[10] Park HW, Choe J, Kang JM. Pressure behavior of transport in fractal porous media using a fractional calculus approach. Energy Source 2000;22:881-90.[11] Raghavan R. Fractional derivatives: application to transient flow. J Petrol Sci Eng 2011;80:7-13.

[12] Cafagna D. Fractional calculus: a mathematical tool from the past for present engineers [past and present. IEEE Trans Ind Electron 2007;1:35-40. [13] Li C, Deng W. Remarks on fractional derivatives. Appl Math Comput 2007;187:777-84.

[14] Gutierrez RE, Rosario JM, Machado JT. Fractional order calculus: basic concepts and engineering applications. Math. Prob. Eng. 2010;2010:19. Article ID375858.

[15] Ortigueira MD. Fractional calculus for scientists and engineers. Netherlands: Springer; 2011.

[16] Herrmann R. Fractional calculus: an introduction for physicists. World Scientific Publishing Company; 2011.[17]

Warren JE, Root PJ. The behavior of naturally fractured reservoirs. SPE J 1963;3:245-55.

[18] Zhao Y, Zhang L. Solution and type curve analysis of fluid flow model for fractal reservoir. World J Mech 2011;1:209-16.

[19] van Everdingen AF, Hurst W. The application of the Laplace transformation to flow problems in reservoirs. JPT J Pet Technol 1949;1:305-24.

[20] Agarwal RG, Al-Hussainy R, Ramey Jr HJ. An investigation of wellbore storage and skin effect in unsteady liquid flow: I. Analytical treatment. SPE J 1970;10:279-90.

[21] Stehfest H. Algorithm 368: numerical inversion of Laplace transforms [D5]. Commun ACM 1970;13:47-9.

[22] Gringarten AC, Bourdet DP, Landel PA, Kniazeff VJ. A comparison between different skin and wellbore storage type-curves for early-time transientanalysis. In: SPE Annual Technical Conference and exhibition, 23-26 September 1979, Las Vegas, Nevada. 